\title{
Adaptive regulation of riboflavin transport in heart: effect of dietary riboflavin deficiency in cardiovascular pathogenesis
}

\author{
Tamilarasan Udhayabanu ${ }^{1}$, Perumal Varalakshmi ${ }^{2}$, Andreea Manole ${ }^{3}$, Henry Houlden ${ }^{3}$ \\ and Balasubramaniem Ashokkumar ${ }^{1 *}$ \\ ${ }^{1}$ Department of Genetic Engineering, School of Biotechnology, Madurai Kamaraj University, \\ Madurai - 625021, INDIA \\ ${ }^{2}$ Department of Molecular Microbiology, School of Biotechnology, Madurai Kamaraj \\ University, Madurai - 625021, INDIA \\ ${ }^{3}$ Department of Molecular Neuroscience and Neurogenetics Laboratory, UCL Institute of \\ Neurology, Queen Square, London, WC1N 3BG, UK
}

*Corresponding Author

Balasubramaniem Ashokkumar

E.mail: $\underline{\text { rbashokkumar@yahoo.com }}$ 


\begin{abstract}
Background:

Riboflavin (Vitamin-B2) is the precursor of coenzymes FAD and FMN that are involved in most of the oxidation-reduction reactions. Especially, in one carbon metabolism FAD serves as a cofactor for MTHFR that regulates remethylation of homocysteine. Elevated homocysteine levels have been implicated in cardiovascular diseases and various types of cancers. Also, studies have documented that riboflavin deficiency leads to mitochondrial dysfunction and apoptosis by altering mitochondrial gene expression and enzymatic levels. However, very little is known about mechanism(s) and regulation of RF uptake in the cardiomyocytes.
\end{abstract}

\title{
Objectives:
}

we aimed to understand the expression levels of riboflavin transporters (rRFT1 and rRFT2) in rat during riboflavin deficiency in heart. In this study we also aimed to explore the in vivo role of riboflavin in ischemic cardiomyopathy and mitochondrial dysfunction based on the expression of cardiac biomarkers, histopathological observations of cardiac tissues and the stability of mitochondrial membrane potential.

\section{Results and Conclusions:}

Functional characterization of riboflavin transporters (RFTs) by riboflavin uptake in different $\mathrm{pH}$ and ions conditions revealed the existence of RFTs in heart. Further, riboflavin deprivation resulted in over expression of both RFTs and lead to cardiovascular diseases due to the accumulation of cardiac biomarkers and reduced mitochondrial membrane potential. Thus, this study addressed the key role of riboflavin in the development of cardiovascular pathogenesis. Besides, the adaptive regulation of riboflavin transporters upon riboflavin deficiency signifies that RFTs can be considered as an effective delivery system for drugs against cardiac diseases. 


\section{Introduction}

Riboflavin (RF) otherwise known as vitamin B2 is one of the water soluble vitamin essential for various flavoenzymes involved in oxidation-reduction reactions by means of its cofactors, flavin mononucleotide (FMN) and flavin adenine dinucleotide (FAD) (Barquera et al., 2002 and Lienhart et al., 2013). Deficiency of RF leads to irregularities in biochemical pathways and causes diseases like, neurological disorders (Cooperman \& Lopez, 1984), cataract (Cumming et al., 2000), various cancers, etc. However, vertebrates are devoid of the capability to synthesis such a crucial micro nutrient. Although gut microflora synthesis riboflavin in minimal quantity, it cannot subsidize the requirement of riboflavin by vertebrates, hence the major source of RF is by dietary intake, which is primarily absorbed in the intestine transporters (RFT/RFVT). As soon as riboflavin enters the blood stream, it is transported to each organ by means of tissue specific RFVTs. Hitherto, three genes, SLC52A1/hRFVT-1, SLC52A2/hRFVT-2 and SLC52A3/hRFVT-3 have been identified and characterized to transport riboflavin in human (Yonezawa et al., 2008; Yao et al., 2010; Subramanian et al., 2011 and Yoshimatsu et al., 2013), whereas in rat only two RFTs have been characterized (Yonezawa et al., 2008; Yamamoto et al., 2009 and Fujimura et al., 2010). The ortholog rRFT-1 (earlier reported by Ericsson et al., 2003 as human ortholog of the porcine receptor for endogenous retrovirus A) in rat was found to be $81.41 \%$ similarity with hRFVT-2 and rRFT-2 was found to be $75.97 \%$ similarity with hRFVT-3. Besides the deficiency of riboflavin, mutations associated with any of these transporters may also lead to diseased conditions by altering the vitamin transport physiology (Green et al., 2010; Johnson et al., 2010; Johnson et al., 2012; Haack et al., 2012). Thus, RFVTs are crucial for transporting and maintaining RF homeostasis in the body. 
As RF is involved in various biochemical pathways, its deficiency may lead to accumulation of unusual or lethal metabolic products. For instance, homocysteine, an atypical amino acid, which is involved in the pathogenesis of Parkinson's and Alzheimer's diseases (Duan, 2002 and Kruman, 2000) is also considered as a risk for cardiovascular diseases (Clarke et al., 1991, Stampfer et al., 1992, Boushey et al., 1995 and Wang et al., 2011). Homocysteine is produced during the synthesis of an essential amino acid methionine in one carbon metabolism. MTHFR (10-methylenetetrahydrofolate reductase) is one of the flavin dependent enzyme that require FAD for the conversion of $\mathrm{N}^{5}, \mathrm{~N}^{10}$-methylenetetrahydrofolate to $\mathrm{N}^{5}$ methyltetrahydrofolate with subsequent conversion of homocysteine to methionine in remethylation pathway of methionine. Furthermore, homocysteine along with reactive oxygen species (ROS) produced during cell division, exert toxic effects in mitochondria by altering mitochondrial membrane potential and permeability (Reid et al., 1966; Ratter et al., 1999 and Lassus et al., 2002). Hence, RF deficiency results in accumulation of homocysteine with subsequent alteration of membrane potential and causes cardiovascular abnormalities. Likewise, dietary RF and MTHFR deficiency in mice during pregnancy resulted in prominent increases the incidence of congenital abnormalities with decreased left ventricular wall thickness in embryonic hearts (Chan et al., 2010). On the other hand, cardioprotective effects of RF against diabetic cardiomyopathy in rats have been demonstrated by reduced formation of lipid peroxides and carbonyl content and also by the increased production of antioxidant enzymes like superoxide dismutase (SOD) and heme oxygenase (HO) (Wang et al., 2011).

The heart contains high levels of RF (Schaus and Kirk, 1955), which is known to be essential for its normal cellular physiology, structure and function. However, cardiac muscle cells in mammals are devoid of the ability to synthesize RF and are dependent on exogenous 
dietary sources through transport across cell membrane. Despite the importance of RF, there is nothing much known about the molecular mechanisms that regulate the RF uptake process and about the distribution of RF transporters in cardiovascular system. In this study, we have aimed to understand the expression pattern of RFVTs in cardiomyocytes using cultured rat cardiomyoblast H9C2 cells and its consequences upon RF deficiency in in vivo conditions with wistar rats as an animal model. In addition, the molecular mechanisms behind the regulation of homocysteine levels and alteration of membrane potential by RF was determined and correlated with mitochondrial dysfunction in cardiomyocytes.

\section{Materials and Methods}

\section{Cell culture and maintenance}

H9C2, a cardiomyoblast cell line of embryonic rat ventricle origin obtained from National Centre for Cell Science, Pune, India was cultured and maintained in Dulbecco's Modified Eagle's Medium (DMEM) supplemented with 10\% FBS, glutamine (0.29 g/L), sodium bicarbonate (2.2 g/L), penicillin (100,000 U/L), and streptomycin (10 mg/L). Cells were grown in $75-\mathrm{cm}^{2}$ plastic flasks at $37^{\circ} \mathrm{C}$ in a $5 \% \mathrm{CO}_{2}-95 \%$ air atmosphere with media changes at every 2 days. Experiments were performed using cells between passages 10 and 16 . H9C2 cells were subcultured by trypsinization with $0.05 \%$ trypsin and 0.9 mM EDTA in PBS free from $\mathrm{Ca}^{2+}$ and $\mathrm{Mg}^{2+}$ and plated onto 24 well plates at a density of $1 \times 10^{5}$ cells/well. Uptake of RF was performed 2-3 days following confluence.

\section{Animal handling and maintenance}

All the animal experiments were performed in accordance with Institutional Ethical Committee of Madurai Kamaraj University, Madurai, India. Standard and RF deficient food pellet was 
purchased from Krish Scientific Shoppe, Bangalore, India. Male wistar rats (150-180 g) were used throughout the study and they were maintained in $25 \pm 2{ }^{\circ} \mathrm{C}$ with an alternating $12 \mathrm{~h}$ dark/light cycle. Initially, during acclimatization period all the animals were fed ad libitum with standard chow (AIN-93M) containing $6.50 \pm 0.42 \mathrm{mg} / \mathrm{kg}$ RF for two weeks. Subsequently, the animals were provided with RF deficient food pellet containing $0.3 \pm 0.05 \mathrm{mg} / \mathrm{kg}$ RF (Fujimura et al., 2010) for 7 weeks, while the animals in control group were provided with standard pellet. Then the experimental animals were euthanized with pentobarbital sodium (50 mg/kg) and organs were collected for further functional characterization.

\section{Riboflavin uptake by cardiomyocytes}

\section{Effect of $\mathrm{pH} \& \mathrm{Na}^{+}$on RF uptake by cells}

RF uptake assay was performed with confluent monolayers of H9C2 cells by incubating with KR buffer containing $\left[{ }^{3} \mathrm{H}\right]$-riboflavin $(6.2 \mathrm{Ci} / \mathrm{mmol}$ with $96 \%$ radiochemical purity was purchased from Moravek Biochemicals, Inc, USA) at $37^{\circ} \mathrm{C}$ for $10 \mathrm{~min}$. Labeled and unlabeled RF were added to the incubation buffer at the onset of uptake experiment and the reaction was terminated after 10 min of incubation by the addition of $2 \mathrm{ml}$ of ice-cold KR buffer followed by immediate aspiration. Cells were then digested by incubating at $80^{\circ} \mathrm{C}$ for 20 min with $1 \mathrm{~N} \mathrm{NaOH}$ followed by neutralization with $\mathrm{HCl}$. Digested cell contents were harvested and then counted for radioactivity using a Scintillation counter (Beckman Coulter LS6500). The protein content of cell digests was measured from the experimental and control wells using Bradford assay and used for the normalization of uptake data. To determine the effect of $\mathrm{pH}$ on RF uptake, the assay was performed with KR buffer adjusted to different $\mathrm{pH}$ ranging from 5.5, 6.5, 7.5 and 8.5. $\mathrm{Na}^{+}$ dependency of RF uptake was tested by isosmotically replacing the $\mathrm{Na}^{+}$in the incubation buffer with other monovalent cation, namely lithium or with the nonionic mannitol. 


\section{Semi-quantitative PCR}

Total RNA from confluent H9C2 cells and cardiac tissue of male wistar rats (100 mg) was isolated using the guanidine isothiocyanate based TRIzol solution (Bio Basic Inc., Canada) according to the manufacturer's specifications. Three micrograms of total RNA were reverse transcribed with oligo(dT) primers using Thermo Scientific DyNAmo cDNA Synthesis Kit (Finnzymes, USA) following the manufacturer's instructions. Semi-quantitative PCR was performed to determine the expression level of rRFT1 and rRFT2 in the H9C2 cells as described previously (Ashokkumar et al., 2007). Gene-specific primers corresponding to the PCR targets were designed by using the standard specifications. For the rRFT1, the primers were forward, 5'CCCGCTGTACAGAGCTTTTC-3’; reverse， 5'-CAGCAGACAGAGGCCATACA-3’. For rRFT2, the primers were forward, 5'- CTGTCACCTTCTTGCCCTTC-3'; reverse, 5'GgtgatACCTGAGCCTTGGA-3'. For GAPDH, the primers were forward, 5'CCATCACCATCTTCCAGGAG-3'; reverse, 5'- CCTGCTTCACCACCTTCTTG-3'. For each

$50 \mu \mathrm{l} \quad$ PCR reaction, $2 \mu \mathrm{l}$ of $\mathrm{cDNA}$ was used as template and the amplification program consisted of 1 cycle at $94^{\circ} \mathrm{C}$ with a 30-s hold followed by $20-30$ cycles at $94^{\circ} \mathrm{C}$ for 1 min, specified annealing temperature with $45-\mathrm{s}, 72^{\circ} \mathrm{C}$ with 30 -s for extension. GAPDH was used as internal control. The PCR samples were electrophoresed on 2.0\% agarose gels in TBE buffer and imaged. Densitometry values were measured at each cycle sampling using the ImageJ software (http://rsb.info.nih.gov/ij). RT-PCR values are presented as a ratio of the specified gene's signal in the selected linear amplification cycle divided by the GAPDH signal.

Western blotting 
Expression of rRFT-1 and -2 proteins in normal and riboflavin deficient rats were examined by western blotting. Fresh heart tissue (100 mg) was minced with RIPA lysis buffer containing (0.01 M Tris-HCl buffer $\mathrm{pH}$ 7.4, 1\% Triton X-100, 1\% sodium deoxycholate, 1\% SDS), supplemented with $1 \mathrm{mg} / \mathrm{ml}$ protease inhibitors, incubated at $4^{\circ} \mathrm{C}$ for $1 \mathrm{~h}$ and homogenized (Rohrbach et al., 1999). Tissue homogenates were centrifuged at 13,000 rpm for 3 min at $4^{\circ} \mathrm{C}$ and supernatant was harvested for protein estimation. Subsequently, $60 \mu \mathrm{g}$ of protein was resolved on $10 \%$ SDS-PAGE. Proteins were transferred to PVDF membrane and the membrane was blocked with 5\% skim milk in Tris-buffered saline (pH 7.4)/0.1\% Tween 20. The membrane was incubated overnight with primary antibodies specific for rRFT-1 (GPR172A rabbit polyclonal antibody - Orb183899, Biorbyt) and rRFT-2 (C20ORF54 rabbit polyclonal antibody SC-85426, Santa Cruz) at a dilution of 1:300 and then washed three times with Tris-buffered saline. Subsequently, the membrane was incubated with secondary antibody coupled with HRP at a dilution of 1:1000 (Goat anti-rabbit IgG-HRP - SC-2004, Santa Cruz) for $1 \mathrm{~h}$. the expression level of rRFT-1 and rRFT-2 was determined based on the immunoreactive bands observed after developing with 3,3'-diaminobenzidine tetrahydrochloride (DAB).

\section{Expression profile of cardiac biomarkers}

Blood sample obtained from the RF deficient rats were checked for the levels of cardiac biomarkers to determine the effect of riboflavin deficiency on cardiomyopathy. Briefly, blood samples were collected from normal and RF deficient rats after 7 weeks of riboflavin deficiency and the well-known cardiac biomarkers viz., homocysteine, creatine kinase, creatine kinase myoglobin in serum samples were measured quantitatively based on the release of NADH using ERBA kits and the chemiluminescence was measured by auto analyzer. While, qualitative assay 
was performed to detect the presence of troponin-I based on sandwich immunoassay using rapid diagnostic kit.

\section{Histopathological examinations}

After seven weeks of experimental conditions, animals were euthanized and heart was excised from normal and RF deficient rats to examine the myocardial damage. For histopathological investigations, excised heart was placed in $4 \%$ formaldehyde with $2 \%$ sodium acetate. The tissue was then embedded in paraffin and cut to a thickness of $5 \mathrm{~mm}$. Histopathological tissue sections of heart were stained with hematoxylin and eosin and then observed under light microscope to study for any morphological changes by RF deficiency.

\section{ROS and mitochondrial membrane potential}

Reactive oxygen species (ROS) production was estimated as a measure of cell death using flow cytometer. For this, cells were dissociated from excised heart tissue by means of cell dissociation solution (Sigma, USA) and assessed for the internal ROS production by treating with 2',7'dichlorodihydrofluorescein diacetate (H2DCF-DA) (Sigma, USA) for 30 min. H2DCF-DA a non-fluorescent compound which upon deacetylation and oxidation reaction by ROS produce highly fluorescent DCF (2',7'-dichlorofluorescein). In addition, mitochondrial membrane potential is crucial for the production of ATP during respiratory chain. Hence, the integrity of mitochondrial membrane was evaluated by treating with MitoTracker Deep Red (Thermo Scientific Fisher, USA), mitochondria-selective probe for 15 min. A total of 10,000 cells were used to assess the ROS production and membrane potential based on the fluorescence emission using flow cytometer (BD FACSAria III, BD Biosciences, USA). FlowJo software was used for data analysis and to discriminate the cell cycle phases. 


\section{Statistical analysis}

Kinetic parameters of the saturable component of RF uptake were calculated by subtracting the diffusing component [determined from the slope of the uptake line between a high substrate concentration of RF (1 mM) and the point of origin] from total uptake at each concentration using Michaelis-Menten equation as described previously (Ashokkumar et al., 2006).

\section{Results}

Functional characterization of riboflavin uptake by cardiomyocytes

\section{Effect of $\mathrm{pH}, \mathrm{Na}^{+}$and extracellular $\mathrm{RF}$ levels on its uptake by cells}

Experiments were performed to understand and validate the potential of cardiomyoblast cell line (H9C2) for RF transport across the membrane using radiolabeled RF $\left({ }^{3} \mathrm{H}-\mathrm{RF}\right)$. Initially, we investigated $\mathrm{pH}$ dependency of cardiomyocytes in RF transport by performing RF uptake assay using ${ }^{3} \mathrm{H}-\mathrm{RF}$ in KR buffer with different $\mathrm{pH}$ ranging from 5.5 to 8.5 and varying ions. The results showed RF (7.5 nm) uptake to be $\mathrm{pH}$ dependent as maximum of RF uptake was at $\mathrm{pH} 7.5$ and started to decline with either increasing or decreasing the $\mathrm{pH}$ to acidic and alkaline conditions. At acidic pH 5.5, 77\% RF uptake rate was lost and 24\% RF uptake rate was lost in alkaline pH 8.5 when compared with $\mathrm{pH} 7.5$ (Fig. 1A). In addition, the effect of ${ }^{3} \mathrm{H}-\mathrm{RF}$ uptake by H9C2 was studied by replacing sodium ion with equimolar concentrations of lithium chloride and mannitol, no changes in the RF uptake was noticed (Fig. 1B), which demonstrated that $\mathrm{Na}^{+}$is not essential for driving riboflavin uptake. Thus, the results revealed that $\mathrm{RF}$ uptake in cardiomyocyte is $\mathrm{pH}$ dependent and sodium independent.

In another study, we examined the initial rate of RF uptake by H9C2 cells as a function of substrate concentration in the KR buffer with $\mathrm{pH} 7.5(0.0075-4.0 \mu \mathrm{M})$. As shown in the Figure 
1C, the results showed RF uptake was concentration dependent and saturable. The apparent $\mathrm{K}_{\mathrm{m}}$ and $\mathrm{V}_{\max }$ of the saturable component of RF uptake were then determined as described in Methods and found to be $3.746 \mu \mathrm{M}$ and $56.391 \mathrm{pmol} / \mathrm{mg}$ protein/10 min, respectively. Thus, it is evident from these results that RF uptake in H9C2 cells is a carrier mediated process in nature.

\section{Effect of riboflavin deficiency in vivo}

After 7 weeks of RF deprivation, the experimental animals were examined for body weight and heart weight. As a result, the body weight and heart weight of male wistar rats fed with RF deficient diet was $190 \pm 10 \mathrm{~g}$ and $0.81 \pm 0.1 \mathrm{~g}$ while the body weight of normal wistar rat was found to be $210 \pm 15 \mathrm{~g}$ and $0.86 \pm 0.1 \mathrm{~g}$ respectively. These results denoted that RF deprivation in rats showed a significant reduction in the final body weight $(P<0.05)$ than control, while changes were not significant with the heart size between the groups. Plasma concentrations of RF was significantly lower in the rats fed with RF deficient diet (2.4 $\mu \mathrm{g} / \mathrm{dl})$ than the control (4 $\mu \mathrm{g} / \mathrm{dl})$

\section{RF transporters expression in the cardiomyocyte H9C2 cells and rat heart}

Rodents are known to contain two RF transporters RFT-1 and RFT-2, where RFT-1 is orthologous to hRFVT-1 and hRFVT-2; RFT-2 is orthologous to hRFVT-3. Thus, to further enhance our understanding the molecular mechanisms responsible for RF uptake, we initially performed semi-quantitative PCR to determine the expression levels of RF transporters in the H9C2 cell lines. The results show that both the transporters rRFT-1 and rRFT-2 were expressed at mRNA levels in cardiomyocytes. In comparison, the basal level expression of rRFT-1 mRNA was four times higher than rRFT-2 mRNA in H9C2 cell line (Fig. 2A). we next investigated the effect of RF deficiency in vivo in the whole heart tissue of rats fed on a RF-deficient chow for 7 weeks compared to controls. As a result, due to riboflavin deprivation the expression of rRFT-1 
and rRFT-2 mRNA was 0.9 and 1.1 fold higher in cardiac tissue of RF deficient rats than controls (Fig. 2B).

In addition to mRNA expression, the expression of RF transporters at protein level was also assessed by western blotting, which revealed that both rRFT-1 and rRFT-2 proteins were expressed in the rat heart tissues (Fig. 3). Albeit, the expression of both rRFT-1 and rRFT-2 was higher at protein levels in cardiac tissue of RF deficient rats compared to normal rats, expression of rRFT-1 was significantly higher compared to rRFT-2 (Fig. 3). Thus, it is evidently observed that RF uptake process is adaptively regulated in the rat heart based on the extracellular RF levels, where the both RF transporters play a major role in such regulation in the cardiac tissue.

\section{Expression profile of cardiac biomarkers}

Having understood the obvious changes in the expression pattern of RF transporters in the heart during RF deficiency, we were intended to underscore any adverse effects in the heart function by RF deficiency. For this, blood sample was collected from normal and RF deficient rats and the level of expression of cardiac biomarkers in the serum samples were determined. the level of homocysteine, creatine kinase and creatine kinase myoglobin were 1.3, 1.4 and 2.0 fold higher in RF deficient rats compared to normal rats (Table 1). Likewise, qualitative analysis performed to check the presence of Troponin-I in RF deficient rat using specific strip showed a band in the test regionThere was no band in the strip containing serum of control rats (Fig. 4), thus showing the increase of Troponin-I levels in RF deficient rats. Thereforethe elevated levels of gold standard cardiac biomarkers that were recorded in RF deficient rat is indicative of cardiac injury in the animal model by RF deficiency.

\section{Histopathological studies}


Next, we investigated whether any gross anatomical changes are induced during RF deficiency by histology with the heart excised from normal and deficient rats by examining under light microscope after staining with hematoxylin-eosin. The results showed aberrations such as degeneration of muscle fibers, loss of cross striations and dwindle nuclei in heart tissue of RF deficient rats, while in control rats the elongated nuclei and intercalated discs were clear (Fig. 5). Therefore, histological examinations suggested that RF deficiency resulted in disruption of the cellular architecture of cardiac cells.

\section{ROS and mitochondrial membrane potential}

To determine whether RF deficiency leads to cardiac dysfunction, the level of ROS and the integrity of mitochondrial membrane potential were investigated by flow cytometry. As shown in the Fig. 6, a shift or reduction in fluorescent intensity in FACS revealed that heart mitochondrial membrane potential and ROS production was reduced in RF deficient conditions. In comparison, a significant reduction in mitochondrial membrane potential was observed in deficient rats (80\%) than control (98\%) (Fig. 6A). Also, a decrease in ROS production was observed in deficient rats (0.9\%) compared to control rats (10\%) (Fig. 6B). Hence, these results collectively illustrated that RF deficiency resulted in cardiac myopathy in rats in vivo.

\section{Discussion}

Riboflavin regulates cellular processes by means of FMN and FAD, which catalyze various biological reactions of carbohydrate, protein, lipid and other vitamin metabolism. Distribution of RFVTs and its mechanism behind RF transport have been characterized in different organs like, liver, kidney, intestine, retina, brain. Heart is another important organ, which serves as body's circulatory pump, wherein RF deficiency complications act as one of the leading cause of 
cardiovascular diseases. Cardiovascular diseases that arise due to unhealthy diet, obesity, use of tobacco, alcohol, etc., are the major cause of death globally. According to WHO 2012, 17.5 million people died from CVD worldwide. But then the precise mechanism behind riboflavin transport and its regulations were poorly understood. This study revealed that the optimum physiological $\mathrm{pH}$ for riboflavin transport by cardiomyocytes is 7.5 and the transport process is $\mathrm{Na}^{+}$independent since there is no effect on the replacement of $\mathrm{Na}^{+}$ion. Thus, RF transport mechanisms in cardiomyocytes were reasonably similar to the mechanism of RF transport by HK-2 cells (Kumar et al., 1998), BeWo cells (Huang and Swaan, 2001), ARPE-19 (Said et al., 2005) and primary hepatocyte of mice (Yao et al., 2013).

Functional characterization of RFTs in rat demonstrated that rRFT-1 is mainly distributed in intestine and able to transport RF in minimal amount (Yonezawa et al., 2008), while rRFT-2 is a major intestinal RF transporter predominantly involved in RF absorption from diet (Yamamoto et al., 2009 and Fujimura et al., 2010). A significant increase in the rRFT-1 expression at mRNA level indicates that rRFT-1 is the predominant transporter in cardiomyocyte H9C2 cells compared to rRFT-2. However a profound increase in the expression of both rRFT-1 and rRFT-2 was observed in RF deficient conditions in the rat heart tissue in vivo. Furthermore, elevated expression of rRFT-1 and rRFT-2 at protein level during RF deficiency was also demonstrated consistently. Overall, these results clearly evidenced that RF uptake process is adaptively regulated in the rat heart depending upon the extracellular RF concentrations, where both rRFT-1 and rRFT-2 plays a major role in such regulation in the cardiac tissue, which appears to be mediated (in part) via transcriptional regulatory mechanisms.

Riboflavin by means of FAD regulates the catabolic pathway of homocysteine, which is well known as a noxious amino acid. RF deficiency was previously shown to elevate the level of 
homocysteine (Lakshmi et al., 1990) which ultimately leads to cardiac dysfunction. Therefore, apart from homocysteine, creatine kinase, creatine kinase myoglobin and cardiac Troponin-I which are deliberated as an early sensitive diagnostic marker for acute myocardial infraction (Adams et al., 1993) were evaluated in this study. As a result, the increased level of cardiac biomarkers such as homocysteine, creatine kinase, creatine kinase myoglobin and Troponin-I than its normal range in the course of RF deficiency suggest that male rats fed with RF deficient feed were found to have impaired cardiac functions and resulted in cardiomyopathy. Subsequently, abnormalities witnessed in the texture of RF deficient cardiac muscle could be due to the accumulation of cardiac biomarkers which further altered the normal pathophysiology of cardiac tissues like ventricular and coronary dysfunction, degeneration of muscle fiber, loss of distinct nuclei, muscle contraction efficiency, etc.,

Cardiomyocytes require high energy for its contraction which can be obtained from the ATP generated in mitochondria. Hence, the integrity of mitochondrial membrane potential is essential for the constant production and supply of ATP. However, the decrease in mitochondrial membrane potential in RF deficient cardiac tissue could be associated with mitochondrial dysfunction and apoptosis due to irregularities in the cardiac profile. Several studies have shown that homocysteine induces ROS production and leads to apoptosis (Sipkens et al., 2007). On the contrary, in some cases homocysteine is proved to regulate the production of ROS differentially in each organ based on the physiology (Forner et al. 2009). For instance, homocysteine was found to increase the level of ROS in kidney mitochondria and reduce it in rat liver, brain and heart mitochondria (Gomez et al., 2011). Thus, the reduced ROS production observed in RF deficient cardiac tissue suggests that homocysteine does not necessarily induce ROS production for the cells to undergo apoptosis. 
Riboflavin transporters seem to have a key role in maintaining riboflavin homeostasis in heart and may be suitable targets for for various drugs with therapeutic potential against cardiovascular diseases. Administration of drugs through GPCR is an effective method than other drug delivery strategies, as they are transducers of extracellular signals and modulators of intracellular signaling. Previously, vitamin transporter like Vitamin B9 (Lu et al., 2002) and B12 (Russell-Jones et al., 1995) were utilized in drug delivery for numerous diseases and were shown to enhance the absorption of drugs conjugated to vitamins. Since, rRFT-1 is a GPCR with crucial role in heart, it can serve as an possible drug target for drugs like prostaglandins, pyrvinium pamoate for treating congestive heart failure and acute myocardial infarction by conjugating with riboflavin, which can able to pass specifically through RFTs across the membrane. Likewise, rRFT-2 deliberated as an ortholog of hRFVT-3 can also be utilized as a therapeutic options for cardioprotective and cardiorestorative treatments.

In conclusion, the results in this study serve as an evidence for the presence of two RF transporters (rRFT1 and rRFT2) in cardiac tissue in the rat. In addition, we have shown that both riboflavin transporters are adaptively regulated upon RF deficiency. We also showed that the deprivation of RF resulted in the accumulation of cardiac biomarkers and the hallmark of congenital heart disease by means of altered cardiac pathophysiology and altered mitochondrial membrane potential. As a consequence, it is clear that supplementation of RF would be beneficial to alleviate the pathologic complications in the heart. Moreover from a pharmaceutical perspective, RF transporters could serve as one of the promising route for target specific drug delivery in to the cells and tissues for treatment against cardiovascular diseases.

\section{Conflict of interest}

Authors have no conflict to declare in this research. 


\section{Acknowledgements}

BA acknowledges DBT-RGYI, INDIA for the financial support [No.BT/PR15134/GBD/27/330/2011] and ICMR, India for the International Biomedical Scientists Fellowship (No.INDO/FRC/452/(Y83)/2016-17-IHD). TU thank DST-PURSE, MKU for the fellowship. Authors gratefully acknowledge DST-PURSE Program (India), Madurai Kamaraj University for the infrastructure and facilities. 


\section{Reference}

1. Barquera B, Zhou W, Morgan JE, Gennis RB. Riboflavin is a component of the $\mathrm{Na}+-$ pumping NADH-quinone oxidoreductase from Vibrio cholerae. Proc Natl Acad Sci U S A. 2002; 99(16):10322-10324.

2. Lienhart W-D, Gudipati V, Macheroux P. The human flavoproteome. Arch Biochem Biophys. 2013; 535 (2):150-162.

3. Cooperman JM, R. Lopez. Riboflavin. In: Handbook of Vitamins: Nutritional, Biochemical and Clinical Aspects, edited by L. J. Machlin. NewYork: Dekker, 1984, 299-327.

4. Cumming RG, Mitchell P, Smith W. Diet and cataract: the Blue Mountains Eye Study. Ophthalmology. 2000; 107 (3):450-56.

5. Yonezawa A, Masuda S, Katsura T, Inui K. Identification and functional characterization of a novel human and rat riboflavin transporter, RFT1. Am. J. Physiol. Cell. Physiol. 2008; 295: 632-641.

6. Yao Y, Yonezawa A, Yoshimatsu H, Masuda S, Katsura T. Identification and Comparative Functional Characterization of a New Human Riboflavin Transporter hRFT3 Expressed in the Brain ${ }^{1-3}$. The Journal of Nutrition Biochemical, Molecular, and Genetic Mechanisms. 2010: $1220-1226$.

7. Subramanian VS, Subramanya SB, Rapp L, Marchant JS, Ma TY, Said HM. Differential expression of human riboflavin transporters $-1,-2$, and -3 in polarized epithelia: A key role for hRFT-2 in intestinal riboflavin uptake. Biochim Biophys Acta Biomembr. 2011; 1808 (12):3016-3021.

8. Yoshimatsu H, Yonezawa A, Yao Y, et al. Functional involvement of RFVT3/SLC52A3 in intestinal riboflavin absorption. Am J Physiol Gastrointest Liver Physiol. 2014; 306 (2):10210.

9. Yamamoto S, Inoue $\mathrm{K}$, Ohta $\mathrm{KY}$, et al. Identification and functional characterization of rat riboflavin transporter 2. J Biochem. 2009; 145 (4):437-443.

10. Fujimura M, Yamamoto S, Murata T, et al. Functional characteristics of the human ortholog of riboflavin transporter 2 and riboflavin-responsive expression of its rat ortholog in the small intestine indicate its involvement in riboflavin absorption. $J$ Nutr. 2010; 140 (10):1722-1727. 
11. Green P, Wiseman M, Crow YJ, et al. Brown-Vialetto-Van Laere Syndrome, a Ponto-Bulbar Palsy with Deafness, Is Caused by Mutations in C20orf54. Am J Hum Genet. 2010; 86 (3):485-489.

12. Johnson JO, Gibbs JR, Van Maldergem L, Houlden H, Singleton AB. Exome sequencing in Brown-Vialetto-van Laere syndrome. Am J Hum Genet. 2010; 87 (4):567-9-70.

13. Johnson JO, Gibbs JR, Megarbane A, et al. Exome sequencing reveals riboflavin transporter mutations as a cause of motor neuron disease. Brain. 2012; 135 (9):2875-2882.

14. Haack TB, Makowski C, Yao Y, et al. Impaired riboflavin transport due to missense mutations in SLC52A2 causes Brown-Vialetto-Van Laere syndrome. J Inherit Metab Dis. 2012; 35 (6):943-948.

15. Duan W, Ladenheim B, Cutler RG, Kruman II, Cadet JL, Mattson MP. Dietary folate deficiency and elevated homocysteine levels endanger dopaminergic neurons in models of Parkinson's disease. J Neurochem. 2002; 80 (1):101-110.

16. Kruman II, Culmsee C, Chan SL, Kruman Y, Guo Z, Penix L, Mattson, MP. Homocysteine elicits a DNA damage response in neurons that promotes apoptosis and hypersensitivity to excitotoxicity. J Neurosci. 2000; 20 (18): 6920-6926.

17. Clare R, Daly L, Robinson K, Naughten E, Cahalane S, Fowler B, Graham I. Homocysteinemia: An independent risk factor forvascular disease. NEJM. 1991; 324 (17): 1149-1155.

18. Stampfer MJ, Malinow MR,Willett W, et al. A prospective study of plasma homocysteine and risk of myocardial infarction in US physicians. JAMA 1992; 268:877-81.

19. Boushey CJ, Beresford SA, Omenn GS, Motulsky AG. A quantitative assessment of plasma homocysteine as a risk factor for vascular disease. Probable benefits of increasing folic acid intakes. JAMA 1995; 274:1049-57.

20. Wang J, Yuan X, Sun B, Cao Y. Inhibition of lipid peroxidation by enzymatic hydrolysates from wheat bran. Food Technol Biotechnol. 2011; 49 (4):481-488.

21. Reid RA, Moyle J, Mitchell P. Synthesis of adenosine triphosphate by a proton motive force in rat liver mitochondria. Nature. 1996; 212: 257-8.

22. Lassus P, Opitz-Araya X \& Lazebnik Y. Requirement for caspase-2 in stress-induced apoptosis before mitochondrial permeabilization. Science 2002; 297, 1352-1354. 
23. Chan J, Deng L, Mikael LG, et al. Low dietary choline and low dietary riboflavin during pregnancy influence reproductive outcomes and heart development in mice. Am J Clin Nutr. 2010; 91 (4):1035-1043.

24. Schaus R, Kirk JE, Lauresn TJS. The riboflavin content of human aortic tissue. J. Geront. 1955; 10: 170-177.

25. Ashokkumar B, Mohammed ZM, Vaziri ND, Said HM. Effect of folate oversupplementation on folate uptake by human intestinal and renal epithelial cells. Am J Clin Nutr. 2007; 86 (1):159-167.

26. Rohrbach S, Yan X, Weinberg EO, et al. Neuregulin in cardiac hypertrophy in rats with aortic stenosis. Differential expression of erbB2 and erbB4 receptors. Circulation. 1999; 100 (4):407-412.

27. Ashokkumar B, Vaziri ND, Said HM. Thiamin uptake by the human-derived renal epithelial (HEK-293) cells: cellular and molecular mechanisms. Am J Physiol Renal Physiol. 2006; 291(4):796-805.

28. Huang S, Swaan PW, Pharmaceutics D, H CPS, Ohio T, Biophysics S. Riboflavin Uptake in Human Trophoblast-Derived BeWo Cell Monolayers: Cellular Translocation and Regulatory Mechanisms. J Pharmacol Exp Ther 2001; 298 (1):264-271.

29. Lakshmi R, Lakshmi V, Bamji MS. Mechanism of impaired skin collagen maturity in riboflavin or pyridoxine deficiency. J Biosci. 1990; 15 (4):289-295.

30. Adams JE, Abendschein DR, Jaffe AS. Biochemical markers of myocardial injury. Is MB creatine kinase the choice for the 1990s? Circulation. 1993; 88 (2):750-763.

31. Sipkens JA, Krijnen PAJ, Meischl C, et al. Homocysteine affects cardiomyocyte viability: Concentration-dependent effects on reversible flip-flop, apoptosis and necrosis. Apoptosis. 2007; 12 (8):1407-1418.

32. Forneris F, Battaglioli E, Mattevi A, Binda C. New roles of flavoproteins in molecular cell biology: histone demethylase LSD1 and chromatin. FEBS J. 2009; 276: 4304-4312.

33. Gomez J, Sanchez-Roman I, Gomez A, et al. Methionine and homocysteine modulate the rate of ROS generation of isolated mitochondria in vitro. J Bioenerg Biomembr. 2011; 43 (4): 377-386.

34. Lu Y, Low PS, Folate-mediated delivery of macromolecular anticancer therapeutic agents, Adv. Drug Deliv. Rev. 2002; 54: 675-693. 
35. Russell-Jones GJ, Westwood SW, Habberfield AD. Vitamin B12 mediated oral delivery systems for granulocyte-colony stimulating factor and erythropoietin, Bioconjug. Chem. 1995; 6: 459-465. 